\title{
ZBTB7A, a potential biomarker for prognosis and immune infiltrates, inhibits progression of endometrial cancer based on bioinformatics analysis and experiments
}

\author{
Rong Geng ${ }^{1,2,3 \dagger}$, Yuhua Zheng ${ }^{1 \dagger}$, Donghua zhou ${ }^{4}$, Qingdong Li ${ }^{1}$, Ruiman $\mathrm{Li}^{2^{*}}$ and Xiaoling Guo ${ }^{1 *}$ (1)
}

\begin{abstract}
Backgroud: ZBTB protein is an important member of the $\mathrm{C} 2 \mathrm{H} 2$ zinc finger protein family. As a transcription factor, it is widely involved in the transcriptional regulation of genes, cell proliferation, differentiation, and apoptosis. The ZBTB7A has been largely linked to different kinds of tumors due to its diverse function. However, the value for ZBTB7A in uterine corpus endometrial carcinoma (UCEC) is unclear.

Methods: In our work, we assessed the importance of ZBTB7A in UCEC. Firstly, Using Oncomine and Tumor Immunoassay Resource (TIMER) databases to evaluate the expression of ZBTB7A. Secondly, we explored the co-expression network of ZBTB7A through the cBioPortal online tool, Metascape, and LinkedOmics. TIMER was also used to explore the relationship between ZBTB7A and tumor immune invasion, and to detect the correlation between the ZBTB7A and the marker genes related to immune infiltration. Finally, CCK8, migration, ChIP assays were introduced to partly validate ZBTB7A function in endometrial cancer cells.

Results: We found the ZBTB7A expression in TIMER was associated with various cancers, especially UCEC. The decreased expression of ZBTB7A was markedly related to the stage and prognosis of UCEC. Furthermore, ZBTB7A was also related to the expression of various immune markers such as Neutrophils, Dendritic cell, T cell (general), Th1, Th2, and Treg. Finally, we verified that ZBTB7A repressed E2F4 transcription and inhibited cells proliferation and migration. These results indicate that ZBTB7A may play a vital role in regulating immune cell infiltration in UCEC, and is a valuable prognostic marker.

Conclusions: In summary, we demonstrate that ZBTB7A is notably downregulated in UCEC, plays a vital role in regulating immune cell infiltration, possesses diagnostic and prognostic values and attenuates E2F4 transcription and cell proliferation, migration in vitro.
\end{abstract}

Keywords: ZBTB7A, Uterine corpus endometrial carcinoma, Prognostic biomarker, Immune cell infiltration, E2F4

*Correspondence: hayyck@126.com; gxl_gr@163.com

${ }^{\dagger}$ Rong Geng and Yuhua Zheng contributed equally to this work

1 Department of Gynecology, Affiliated Foshan Maternity \& Child Healthcare Hospital, Southern Medical University, Foshan 52800, China

2 Department of Gynecology and Obstetrics, The First Affiliated Hospital, Jinan University, Guangzhou 510632, China

Full list of author information is available at the end of the article

\section{Background}

Uterine corpus endometrial carcinoma (UCEC) is the sixth-most-common type of cancer in females, and the incidence is rapidly increasing in recent years [1]. The 2018 data shows that 382,000 women are diagnosed and 90,000 deaths due to UCEC worldwide [2]. The factors that affect endometrial cancer are diverse.

(c) The Author(s) 2020. This article is licensed under a Creative Commons Attribution 4.0 International License, which permits use, sharing, adaptation, distribution and reproduction in any medium or format, as long as you give appropriate credit to the original author(s) and the source, provide a link to the Creative Commons licence, and indicate if changes were made. The images or other third party material in this article are included in the article's Creative Commons licence, unless indicated otherwise in a credit line to the material. If material is not included in the article's Creative Commons licence and your intended use is not permitted by statutory regulation or exceeds the permitted use, you will need to obtain permission directly from the copyright holder. To view a copy of this licence, visit http://creativeco mmons.org/licenses/by/4.0/. The Creative Commons Public Domain Dedication waiver (http://creativecommons.org/publicdomain/ zero/1.0/) applies to the data made available in this article, unless otherwise stated in a credit line to the data. 
First, the instability of estrogen levels is one of the most common causes, so postmenopausal women are more susceptible to endometrial cancer [3]. Besides, many factors, including smoking, hypertension, overweight, and genetics, play a vital role in the development of endometrial cancer [4]. Despite progress in the treatment of endometrial cancer, endometrial cancer is one of the human malignant diseases for which mortality is increasing. Unexpected recurrences and poor prognosis still confuse clinicians. Therefore, it is an urgent need to detect more therapeutic targets and prognostic markers.

ZBTB7A is also called Pokemon, factor binding IST protein-1(FBI-1), and the lymphoma/leukemia-related factor (LRF). ZBTB7A is overexpressed in many human cancers, including ovarian [5], breast [6], as an oncogene. Further research on the carcinogenic function of ZBTB7A was found that ZBTB7A could affect the survival, proliferation, apoptosis, and migration of cancer cells. Besides, the expression of ZBTB7A is also related to many clinicopathological parameters, including tumor size, histological grade, and overall patient survival. ZBTB7A regulates gene expression by transducing other transcription factor signals and is a widely used as promoter factor [7]. In breast cancer, ZBTB7A controls the expression of ER $\alpha$ by directly binding the GC-rich region in the ER $\alpha$ promoter [8]. In hepatocellular carcinoma, ZBTB7A increases the expression of P53 and initiates caspase-dependent apoptosis through death receptors and mitochondrial pathways [9]. Recent studies have shown that ZBTB7A is closely related to the tumor microenvironment and immune cell infiltration in prostate cancer [10]. It is worth notable that the chromosomal region 19p13.3, including ZBTB7A, is often deleted in human cancer. The loss of ZBTB7A may be one of the significant potential mechanisms that causes cancer [11]. However, the expression pattern and its significance in UCEC have not been well identified.

Our results indicate that ZBTB7A may be a new potential prognostic marker in endometrial cancer. We analysed the expression, mutation and prognosis of ZBTB7A in UCEC patient via Oncomine, GEPIA, TIMER, and cBioPorta database. The relation of ZBTB7A and immune cell infiltration was further investigated through TIMER. Moreover, we expanded discussion to see if the association holds up in pancarcinomas. Finally, biomedical experiments, such as CCK8, migration and ChIP assays were carried out to validate conclusion from bioinformatic analysis partly. Therefore, our results reveal ZBTB7A as a new target and direction for the diagnosis and treatment of endometrial cancer.

\section{Materials and methods}

\section{Oncomine database analysis}

The expression level of the ZBTB7A gene in UCEC was estimated in the Oncomine 4.5 database (https://www. oncomine.org/). Oncomine is a comprehensive database related to cancer assessment. The threshold was determined according to the following values: P-value of 0.01 , fold change of 2 , and gene ranking of all.

\section{GEPIA database analysis}

The Gene Expression Profiling Interactive Analysis (GEPIA) database (https://gepia.cancer-pku.cn/) is an online database that includes 9,736 tumors and 8,587 normal samples from TCGA and the GTEx projects [12]. We applied GEPIA to evaluate the expression and survival of ZBTB7A, including overall survival (OS) and disease-free survival (DFS) based on gene expression with the log-rank test and the Mantel-Cox test in UCEC.

\section{UALCAN database analysis}

UALCAN (https://ualcan.path.uab.edu) uses TCGA level 3 RNA-seq and clinical data from 31 cancer types [13]. UALCAN was used to analyze the relative gene expression of tumor and normal samples, as well as the gene expression of different tumor subgroups. In addition, based on individual cancer stage, tumor grade, or other clinicopathological features, UALCAN can also be used for analysis.

\section{cBioPortal database analysis}

The cBio Cancer Genomics Portal (https://cbioportal .org) has multidimensional cancer genomics datasets [14]. The mutation of ZBTB7A in UCEC was analyzed using the cBioPortal tool in this study.

\section{LinkedOmics database analysis}

The LinkedOmics database (https://www.linkedomics) is mainly used to analyze 32 TCGA cancer-related data sets and is a comprehensive online platform [15]. We analyzed the co-expression of ZBTB7A on the volcano map, heat map, or scatter map through the LinkedOmics function module by using Pearson's correlation coefficient. Besides, we analyzed kinase-target enrichment, miRNA-target enrichment, and transcription factortarget enrichment through gene set enrichment analysis (GSEA). The latter two network analyses were built on the Molecular Signatures Database (MSigDB). The rank norm was $\mathrm{FDR}<0.05$, and 500 simulations were performed.

\section{TIMER database analysis}

TIMER is a comprehensive resource for systematic analysis of immune infiltrates across diverse cancer types from TCGA (https://cistrome.shinyapps.io/timer/), which 
includes 10,897 samples across 32 cancer types [16]. TIMER speculates the abundance value of tumor infiltrating immune cells (TIICs) from the gene expression profile by applying the method of deconvolution [17]. We analyzed ZBTB7A and the correlation of ZBTB7A expression with the abundance of immune infiltrates, including B cells, CD4+ T cells, CD8 $+\mathrm{T}$ cells, neutrophils, macrophages, and dendritic cells in UCEC and other cancers.

\section{STRING database analysis}

STRING database (version 10.5) was acquired proteinprotein interaction information of DEmRNAs (https:// string-db.org/). Edges with weight beyond a threshold of 0.4 were displayed. Hub genes were found by using "barplot" in the R language [18].

\section{Metascape database analysis}

Metascape [19] (https://metascape.org), which was updated in 2018, is a web-based tool that provides gene functional annotation and enrichment analysis. Gene enrichment was analysed from Gene Ontology (GO) and Kyoto Encyclopedia of Genes and Genomes (KEGG) through the database to predict the potential biological value of overlapping genes between target genes and DEGs.

\section{Cell culture}

endometrial cancer cell line Ishikawa cells were cultured with RPMI-1640 Medium (Gibco, Carlsbad, USA) with $10 \%$ fetal bovine serum (Gibco) at a humidified atmosphere of $5 \% \mathrm{CO}_{2}$ at $37^{\circ} \mathrm{C}$.

\section{Cell viability assay}

For CCK8 assay, 1000 ZBTB7A overexpressed and control cells were planted into 96-well plates, Which were cultured for varied days (1, 2, 3 and 4 day). At the point time, CCK- 8 solution (G-Clone, China) $20 \mu \mathrm{l}$ was added to each well and nurtured for another $4 \mathrm{~h}$ at $37{ }^{\circ} \mathrm{C}$. Finally, the optical density (OD) value at $450 \mathrm{~nm}$ was set by micro-plate reader.

\section{Migration assay}

$2 \times 10^{5}$ Ishikawa cells per/well were seeded into the upper chamber of $8 \mu \mathrm{m}$ pore (BD Falcon, USA). Chambers were embeded into correlating 24-well plate containing RPMI1640 medium with $20 \%$ FBS. Cells in the upper chambers were cultured without FBS. After 16-20 h, migrated cells sticking to the lower surface of chamber were fixed and stained with $5 \%$ crystal violet and were calculated in five fields of microscope.

\section{Quantitative PCR}

The experimental procedures were described previously [20]. The primers used were listed as follows: F: 5'-CATCCAGTGGAAGGGTGTG-3'， R: 5'-TTGGAC GTGAGGCTTCCTG-3' (E2F4); F: 5'-TGCAAGGTC CGCTTCACCAG-3', R: 5'-TGCAAGGTCCGCTTC ACCAG-3' (ZBTB7A). F: 5'-CTCCTCCTGTTCGAC AGTCAGC-3', R: 5'-CCCAATACGACCAAATCC GTT-3'(GAPDH). qPCR Parameters were confirmed as below: $95^{\circ} \mathrm{C}$ for $15 \mathrm{~s} ; 95^{\circ} \mathrm{C}$ for $5 \mathrm{~s}, 60^{\circ} \mathrm{C}$ for $40 \mathrm{~s}$ for 40 cycles; $95^{\circ} \mathrm{C}$ for $15 \mathrm{~s}, 60^{\circ} \mathrm{C}$ for $1 \mathrm{~min}$ and $95^{\circ} \mathrm{C}$ for $15 \mathrm{~s}$.

\section{Luciferase assays}

The three deleted potential ZBTB7A binding motif of E2F4 $(-483 \sim-471,-45 \sim-43,+9 \sim+21)$ and full length of E2F4 promoter $(-2000 \sim+100)$ were constructed into pGL3-based vector. Cells were co-transfected with corresponding constructed pGL3 reporter plasmid and pcDNA4-ZBTB7A using Lipofectamine 2000 (Invitrogen, Carlsbad, CA, USA). After transfection $36 \mathrm{~h}$, the cells were collected and measured by Dual-Luciferase Reporter assay (Promega, Madison, WI, USA). All plasmids were synthesized by the Genewiz Company (Suzhou, China).

\section{Chromatinimmunoprecipitation (ChIP) assay}

ChIP assays were performed via ChIP Kit (Beyotime Shanghai, China). Briefly, the protein/DNA complexes were prepared $48 \mathrm{~h}$ from Ishikawa cells. Crosslinking of proteins to DNA was fixed using $1 \%$ formaldehyde for $10 \mathrm{~min}$. The DNA were sheared to $200-1000 \mathrm{bp}$ lengths sonically on ice. The lysates were slow whirling overnight at $4{ }^{\circ} \mathrm{C}$ with anti-ZBTB7A antibody (A300-549A, Bethyl) or Rabbit IgG (B900610, Proteintech). Then the ZBTB7A binding DNA was precipitated and purified. qRT-PCR was employed to analyze the enriched DNA. Primers for E1 element F: AGGACCATGGGTGGA ACAAG, R: CCTAAAGGCTGCCCTACTGTC.

\section{Statistical analysis}

Gene expression data were analyzed using the P-values, fold changes, and ranks from the Oncomine database. Survival curves were produced by the GEPIA database. The correlation of gene expression was evaluated in the TIMER databases using Spearman's correlation analysis. Other data were evaluated using Student's t-test. P value $<0.05$ was considered statistically significant.

\section{Results}

The levels of ZBTB7A mRNA in UCEC and other cancers ZBTB7A transcription levels in UCEC study were evaluated from oncomine and TIMER. The mRNA 
expression and DNA copy number variation (CNV) of ZBTB7A were significantly lower in UCEC tissues than in healthy tissues revealed in the Oncomine 4.5 database ( $\mathrm{P}$ 0.01). ZBTB7A ranked within the top $20 \%$ based on mRNA expression and within the top 0.5\% depended on DNA CNVs when the fold differences were within 2 (Fig. $1 \mathrm{a}-\mathrm{c}$ ). We compared the mRNA expression of ZBTB7A between UCEC and normal tissues of the endometrium. The results indicated that the expression level of ZBTB7A was lower in UCEC than in normal endometrium by the GEPIA dataset (https:// gepia.cancer-pku.cn/) (Fig. 1d, e). Besides, The analysis of TCGA RNA-seq data using the TIMER database showed that ZBTB7A mRNA expression was also markedly downregulated in BLCA (bladder urothelial carcinoma), COAD (colon adenocarcinoma), LUAD (lung adenocarcinoma), READ (rectum adenocarcinoma) tissues in compared with the corresponding normal tissues (Fig. 1f). By contrast, ZBTB7A expression was upregulated in BRCA (breast invasive carcinoma), CHOL (cholangiocarcinoma), HNSC-HPV pos
(Head and Neck squamous cell carcinoma-human papilloma virus positive), HNSC-HPV neg (Head and Neck squamous cell carcinoma-human papilloma virus negative) and LIHC (liver hepatocellular carcinoma). These data implied that downregulated ZBTB7A expression in UCEC might act as a potential diagnostic indicator.

\section{Sub-group analysis of multiple clinic pathological features and survival of ZBTB7A}

Further analysis of various clinic subtype features in 546 UCEC samples from the TCGA consistently established low transcription of ZBTB7A. The ZBTB7A transcription level was markedly lower in UCEC patients than in the healthy endometrium group associated with stages, age, and race (Fig. 2a-c). We further tested the clinical efficiency of ZBTB7A in UCEC. TIMER analyses uncovered that patients with downregulated ZBTB7A mRNA level harbored unfavorable overall survival (OS) ad disease free survival (DFS) $(\mathrm{P}<0.05)$ (Fig. $2 \mathrm{~d}$, e) in UCEC. Besides, we also found that ZBTB7A mRNA expression was related to the survival in 2 cancers out of 9 cancers,

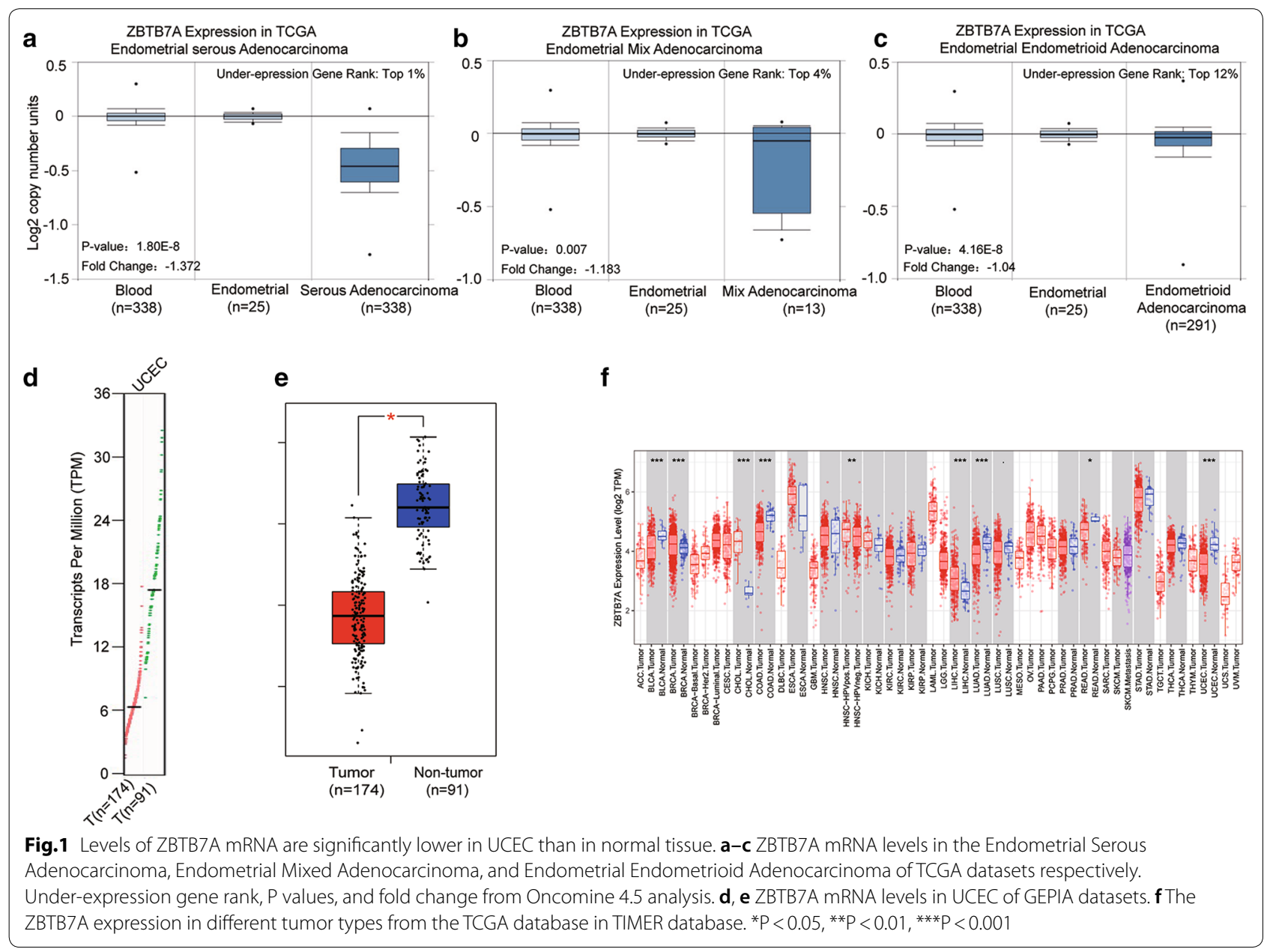


a

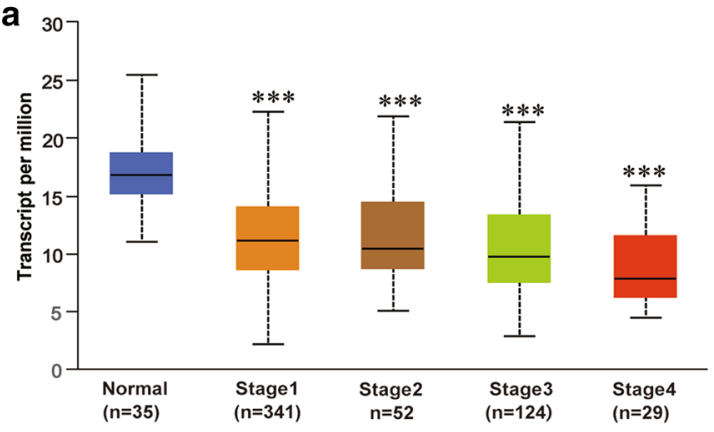

b

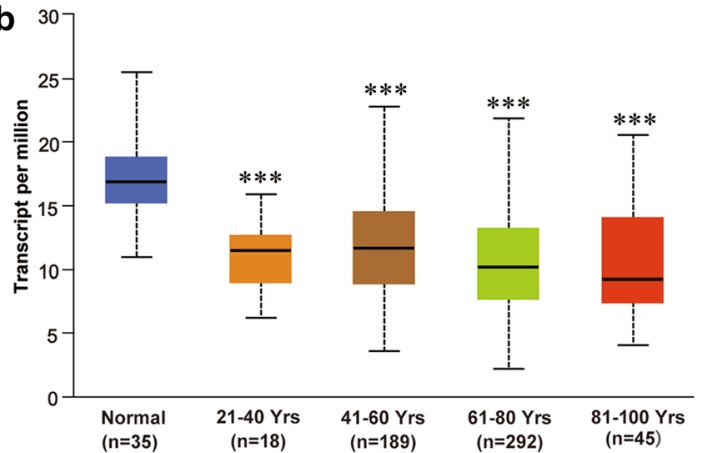

C

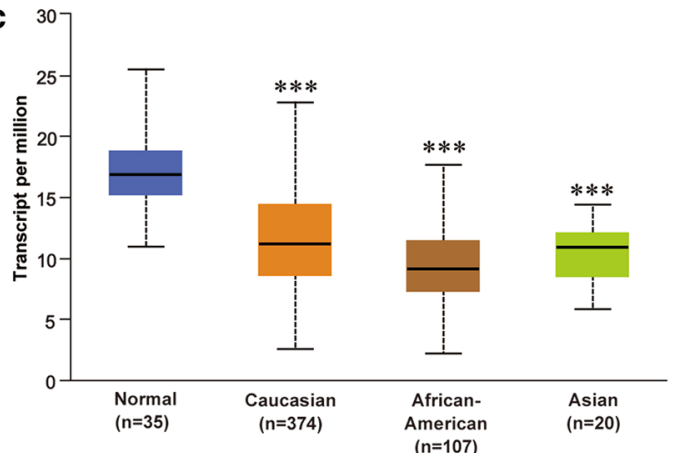

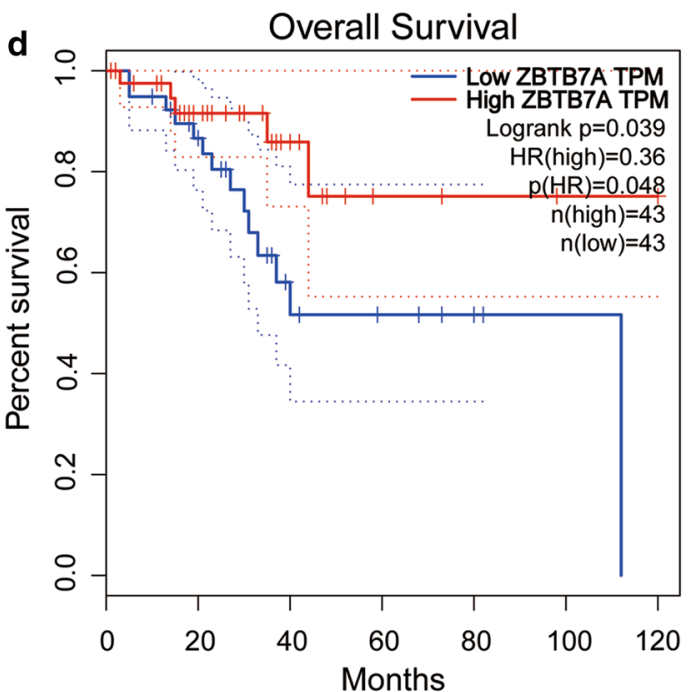

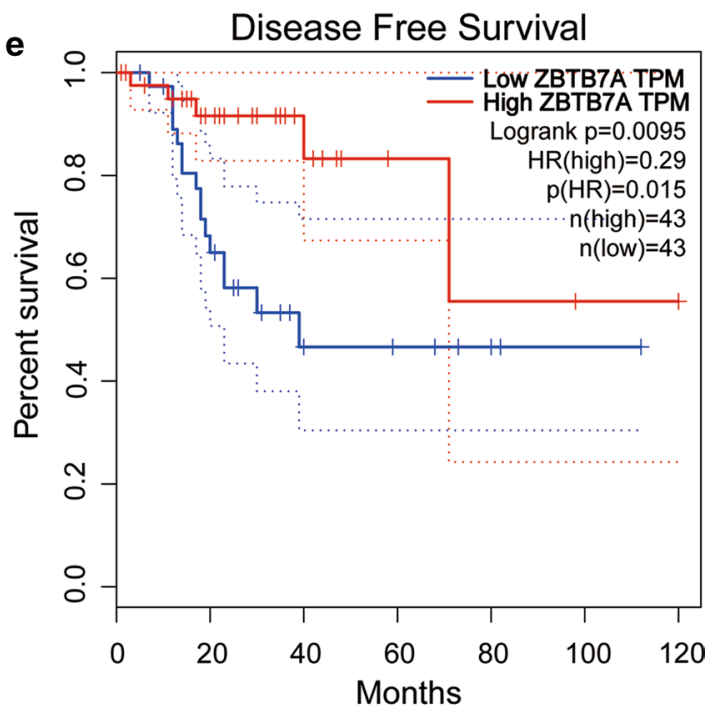

Fig.2 ZBTB7A level in subgroups of patients with UCEC, stratified based on disease stages, age, race (UALCAN) and survival (GEPIA). a Boxplots show relative expression of ZBTB7A in normal individuals or in UCEC patients in stages 1,2, 3 or 4; (b) in normal individuals of any age or in UCEC patients aged 21-40, 41-60,61-80, or 81-100 years; (c) in normal individuals of any ethnicity or in UCEC patients of Caucasian, African-American or Asian ethnicity. $\mathbf{d}$-e Overall survival and disease free curves of ZBTB7A in UCEC in GEPIA

including $\mathrm{CHOL}$ and $\mathrm{HNSC}-\mathrm{HPV}$ pos (Additional file 1: Fig. S1).

\section{ZBTB7A genes mutation and protein interaction} relationship analysis in UCEC.

In this study, the cBioPortal online tool was used for analysis of ZBTB7A alterations. ZBTB7A was altered in 36 samples out of 409 patients with UCEC (9\%). Two or more aberrant changes for ZBTB7A were detected in 19 samples (3.47\%) (Fig. 3a). The protein interaction relationships between key genes were analyzed using STRING, and a strong relationship between critical genes was shown in Fig. 3b.

\section{Enriched genes of UCEC in pathway and process enrichment analysis}

For finding enriched genes in UCEC, pathway and process enrichment analysis were achieved using the following database resource: GO Biological Processes, KEGG Pathway, Canonical Pathways, Reactome Gene 


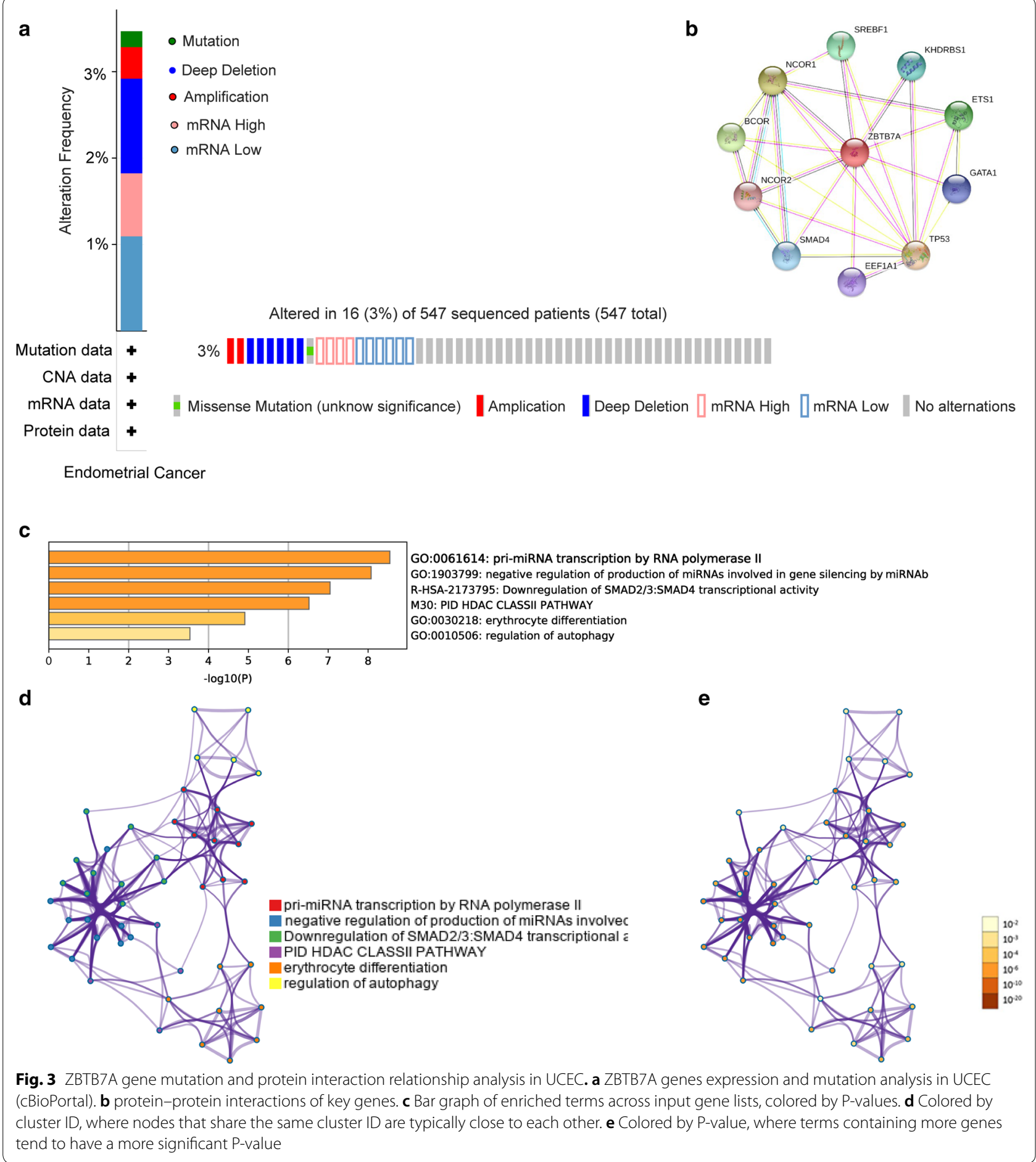

Sets, and CORUM. Top 6 representative enriched clusters were shown in Fig. 3c. To further catch the relationships between the terms, a subset of enriched terms had been selected and displayed as a network plot, where terms with a similarity $>0.3$ were connected by edges.
The network was visualized using Cytoscape, where each node represented an enriched term and colored first by its cluster ID (Fig. 3d) and then by its P-value (Fig. 3e). Specifically, the genes enriched in UCEC involved in several biological functions, such as pri-miRNA 
transcription by RNA polymerase II, negative regulation of production of miRNAs involved in gene silencing by miRNA, erythrocyte differentiation, regulation of autophagy, and so on.

\section{ZBTB7A networks of kinase, miRNA or transcription factor targets in UCEC}

To further explore the targets of ZBTB7A in UCEC, we analyzed the kinase, miRNA and transcription factor target networks of positively correlated gene sets generated by GSEA (Table 1). The top 5 most significant target networks were the kinase-target networks related primarily to the kinases polo like kinase 1 (PLK1), cyclindependent kinase 2 (CDK2), Aurora kinase B (AURKB), cyclin-dependent kinase 1 (CDK1), and tumor-associated macrophages (ATM). In fact, PLK1, CDK1, and AURKB were significantly upregulated in tumor tissues, by contrast, ATM was markedly downregulated expressed in tumor tissues, except CDK2 (Additional file 2: Fig. S2). In addition, all these kinase genes were not significantly associated with the OS of UCEC (Additional file 2: Fig. S2). The miRNA-target network was associated with (GACTGTT) miR-212, miR-132, (AGTCTTA) miR-499, (CTGTTAC) miR-202, (TGTATGA) miR-485-3P, and (GGGATGC) miR-522. The transcription factor-target network was related mainly to the E2F transcription factor (E2F) family, including V\$E2F4DP2_01, V\$E2F_ Q4_01, V\$MAZ_Q6, GCGSCMNTTT_UNKNOWN, and KCCGNSWTTT_UNKNOWN.
TPX2 and TTK in the ZBTB7A co-expression network may be targets of the E2F4 transcription factor

The function module of Linkedomics was used to analyze mRNA sequencing data from 176 UCEC patients in the TCGA. As shown in the volcano plot (Fig. 4a), 8768 genes (dark red dots) showed significant positive correlations with ZBTB7A, whereas 11,130 genes (dark green dots) showed significant negative correlations (false discovery rate $[\mathrm{FDR}]<0.01)$. The heat map indicated that the 50 significant gene sets positively and negatively correlated with ZBTB7A (Fig. 4b, c). Among 50 negatively related genes, The expression of TPX2 (Fig. 4d, e) and TTK (Fig. $4 \mathrm{~g}-\mathrm{h}$ ) are upregulated and related to the poor prognosis of UCEC. The level of TPX2 (Fig. 4f, $R=0.26$, $\mathrm{P}$-value $=0.00057$ ) and TTK (Fig. 4i, $\mathrm{R}=0.29, \mathrm{P}$-value $=$ 0.00014 ) has direct correlation with E2F4 in UCEC. Previous studies showed that in esophageal squamous cell carcinoma, LINC00337 could recruit E2F4 to upregulate TPX2 transcription, form LINC00337/E2F4/TPX2 axis [21]. In anaplastic thyroid cancer (ATC), E2F4 overexpression inhibits the transcription of the TTK in ATC cells [22]. The above suggests that TPX2 and TTK may be the target genes of E2F4 in UCEC.

\section{ZBTB7A is correlated with tumor purity and immune infiltration level in UCEC}

We investigated whether ZBTB7A expression was correlated with immune infiltration levels in UCEC from the TIMER database. The results showed that ZBTB7A expression had no correlations with tumor purity $(\mathrm{r}=$

Table 1 The Kinase, miRNA and transcription factor-target networks of ZBTB7A in UCEC (LinkedOmics)

\begin{tabular}{llcc}
\hline Enriched Category & Geneset & LeadingEdgeNum & FDR \\
\hline Kinase & Kinase_PLK1 & 41 & 0 \\
target & Kinase_CDK2 & 112 & 0 \\
& Kinase_AURKB & 34 & 0 \\
& Kinase_CDK1 & 94 & 0 \\
& Kinase_ATM & 63 & 0 \\
miRNA target & GACTGTT, miR-212, miR-132 & 57 & 0.0091549 \\
& AGTCTTA, miR-499 & 37 & 0.010299 \\
& ATACCTC, miR-202 & 63 & 0.020598 \\
& TGTATGA, miR-485-3p & 57 & 0.020598 \\
Transcription factor target & ACCATTT, miR-522 & 62 & 0.025176 \\
& V\$E2F4DP2_01 & 80 & 0 \\
& V\$E2F_Q4_01 & 69 & 0 \\
& V\$MAZ_Q6 & 52 & 0.00032365 \\
& GCGSCMNTTT_UNKNOWN & 33 & 0.00033906 \\
\hline
\end{tabular}

LeadingEdgeNum, the number of leading edge genes; FDR, false discovery rate from Benjamini and Hochberg from gene set enrichment analysis (GSEA); V\$, the annotation found in Molecular Signatures Database (MSigDB) for transcription factors (TF) 


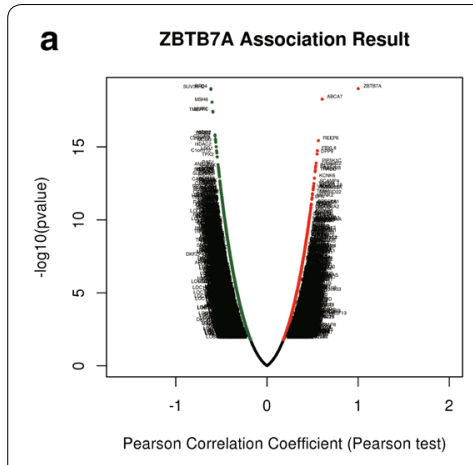

d

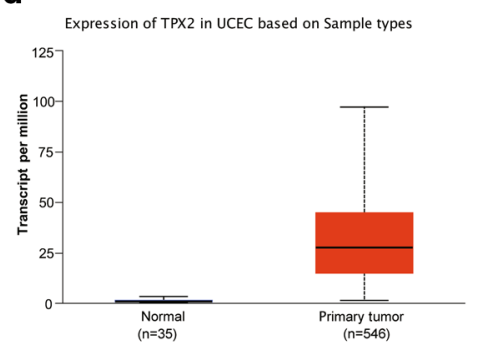

g

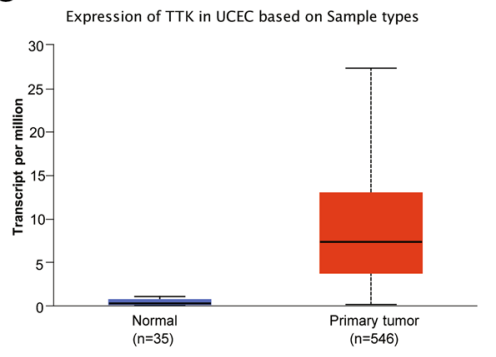

b
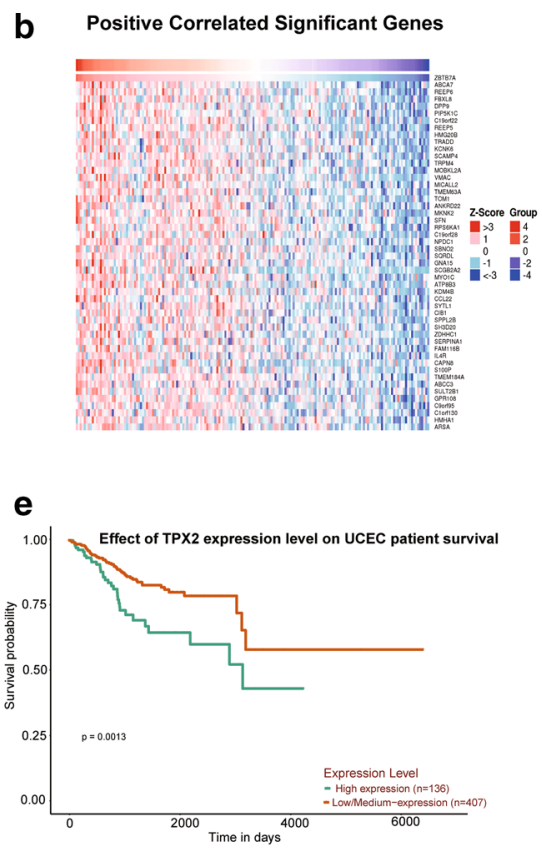

h

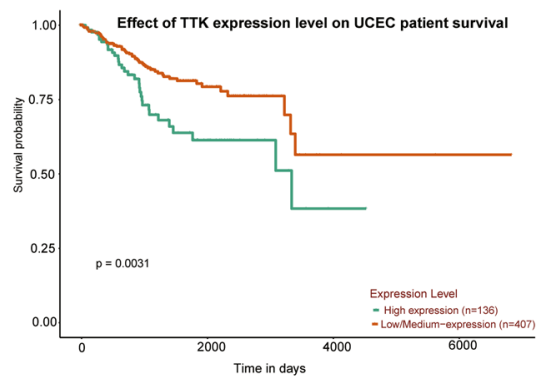

C Negatively Correlated Significant Genes

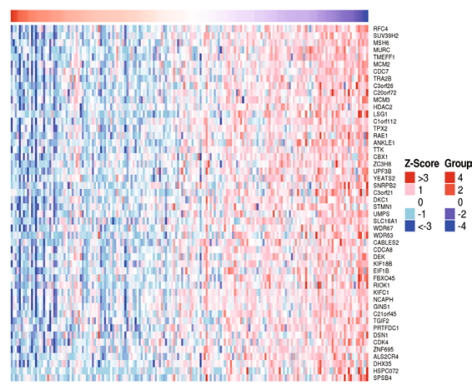

f

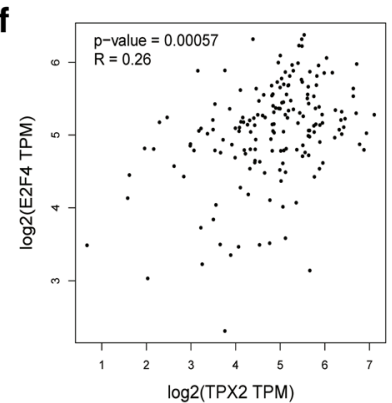

i

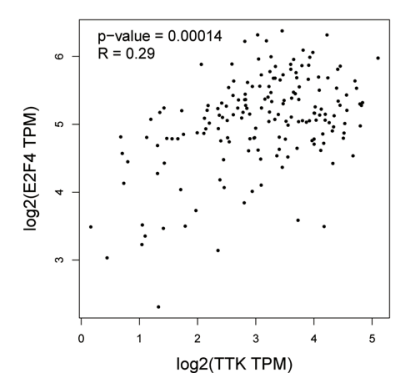

Fig. 4 Genes differentially expressed in correlation with ZBTB7A in UCEC (LinkedOmics). a A Pearson test was used to analyze correlations between ZBTB7A and genes differentially expressed in UCEC. $\mathbf{b}-\mathbf{c}$ Heat maps showing genes positively and negatively correlated with ZBTB7A in UCEC. Red represents positively correlated genes and green indicates negatively correlated genes. The high expression of TPX2 (d, e) and TTK ( $(\mathbf{g}, \mathbf{h})$ demonstrates unfavorable progression in UCEC, and shows positive relativity with E2F4 (f, i)

$-0.016, P=2.01 \mathrm{E}-01$ ), but powerful correlated with the immune cells infiltration levels include CD8 $+\mathrm{T}$ cells, neutrophils, and dendritic cells (Fig. 5a). Besides, we explored the relationship between ZBTB7A expression and the infiltrating immune cells in 9 cancer types, including BLCA, COAD, LUAD, READ, BRCA, CHOL, HNSC-HPV pos, HNSC-HPV neg, LIHC using the TIMER database. The results showed that ZBTB7A expression negatively correlated with tumor purity in 2 cancer types (LUAD, READ). Moreover, ZBTB7A expression significantly correlated with the infiltration levels of B cells in 4 cancer types (BRCA, LIHC, LUAD, and READ), CD4+T cells in 7 cancer types (BRCA, COAD, HNSC-HPV pos, HNSC-HPV neg, LIHC, LUAD, and READ), macrophages in four cancer types (BRCA, CHOC, LIHC, and LUAD), neutrophils in six cancer types (BLCA, CHOL, COAD, HNSC-HPV pos, HNSCHPV neg, and LUAD), dendritic cells in five cancer types (COAD, HNSC-HPV pos, HNSC-HPV neg, LIHC, and LUAD). However, ZBTB7A was not correlated with the infiltration levels of $\mathrm{CD} 8+\mathrm{T}$ cells in nine cancer types (Additional file 3: Fig. S3).

Particularly, ZBTB7A CNV has significant correlations with infiltrating levels of $\mathrm{CD} 8+\mathrm{T}$ cells, macrophages, neutrophils, and dendritic cells (Fig. 5b). Otherwise, ZBTB7A CNV has significant correlations with infiltrating levels of $\mathrm{B}$ cell in 5 cancer types (BRCA, LUAD, COAD, HNSC-HPV pos, and HNSC-HPV neg), CD8+ T 

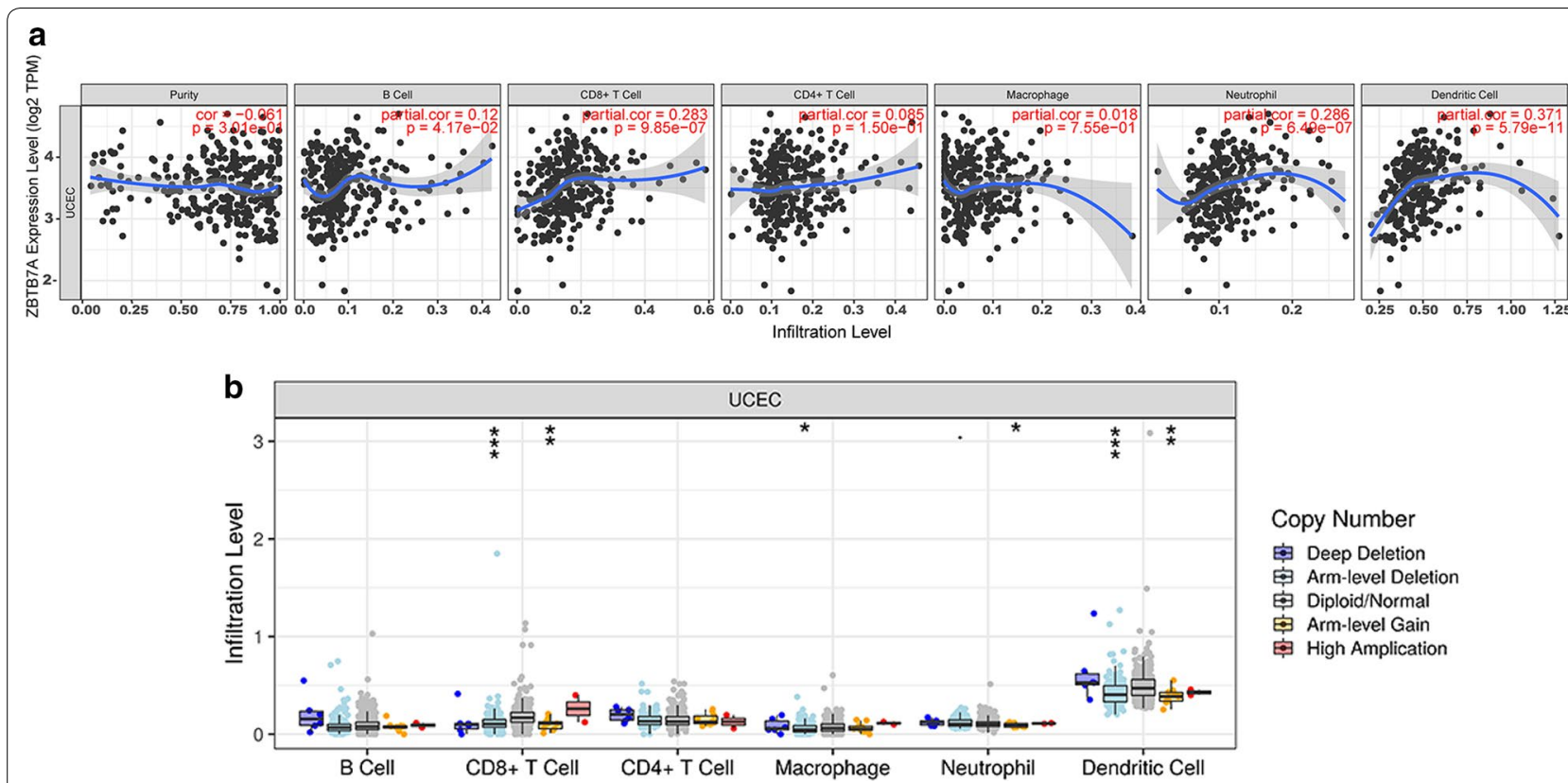

Copy Number

审 Deep Deletion

审 Arm-level Deletion

审 Diploid/Normal

由 Arm-level Gain

审 High Amplication positively correlations with infiltrating levels of $C D 8^{+} T$ cells, Neutrophil and Dendritic cells. b ZBTB7A CNV affects the infiltrating levels of CD8+T cells, Macrophages, Neutrophils, and Dendritic cells in UCEC

cells in 4 cancer types (BRCA, COAD, HNSC-HPV pos, and HNSC-HPV neg), CD4+ T cells in 2 cancer types (BLCA and BRCA), macrophages in 4 cancer types (BLCA, BRCA, LUAD, and HNSC-HPV neg), neutrophils in 6 cancer types (BRCA, CHOL, LUAD, READ, COAD, and HNSC-HPV neg), dendritic cells in 3 cancer types (LUAD, COAD, and HNSC-HPV neg) (Additional file 4: Fig. S4).

\section{Correlation analysis between mRNA levels of ZBTB7A and markers of different subsets of immune cells}

Next, we investigated the relevance between ZBTB7A expression and the status of tumor-infiltrating immune cells based on the levels of immune marker gene in UCEC tissues through investigation the TIMER and GEPIA databases. The immune cells analyzed in UCEC tissues included CD8 + cell, B cells, tumor-associated macrophages (TAMs), neutrophils, and Dendritic cell. Moreover, different subsets of $\mathrm{T}$ cells, namely, $\mathrm{T}$ cell (general), T helper 1 (Th1), Th2, Th17, and regulatory $\mathrm{T}$ (Tregs) were also examined. Tumor purity of clinical samples influences the analysis of immune infiltration, the correlation analysis was adjusted for purity (Table. 2). Data from TIMER databases showed that ZBTB7A in UCEC tissues had a strong correlation with the marker genes from tumor-infiltrating neutrophils, dendritic cell (Fig. 6 and Table. 2). Specifically, ZBTB7A expression domonstrated noticeable interaction with the markers of specific immune cells such as neutrophils marker, CD66b ( $\mathrm{r}=0.309 ; \mathrm{P}=6.87 \mathrm{e}-08), \mathrm{CD} 11 \mathrm{~b}(\mathrm{r}=$ 0.294; $\mathrm{P}=3.01 \mathrm{e}-07)$, and CCR7 $(\mathrm{r}=0.222 ; \mathrm{P}=1.27 \mathrm{e}-$ 04), Dendritic cell markers, HLA-DPB1 $(\mathrm{r}=0.212 ; \mathrm{P}=$ 2.63e-04), HLA-DQB1 $(r=0.134 ; \mathrm{P}=2=\mathrm{e}-02)$, HLADRA $(r=0.255 ; \mathrm{P}=9.82 \mathrm{e}-06)$, HLA-DPA1 $(\mathrm{r}=0.238$; $\mathrm{P}$ $=3.87 \mathrm{e}-05)$, BDCA1 $(\mathrm{r}=0.246 ; \mathrm{P}=2.00 \mathrm{e}-05)$, BDCA4 $(\mathrm{r}=0.226 ; \mathrm{P}=9.25 \mathrm{e}-05)$, and $\mathrm{CD} 11 \mathrm{c}(\mathrm{r}=0.259 ; \mathrm{P}=$ 7.05e-06). Besides, The expression of ZBTB7A also exhibited relevance with the marker genes of different subsets of $\mathrm{T}$ cells, namely, $\mathrm{T}$ cell (general), CD3D $(\mathrm{r}=$ 0.139; $\mathrm{P}=1.72 \mathrm{e}-02)$, Th1 marker, STAT4 $(\mathrm{r}=0.166$; $\mathrm{P}$ $=4.40 \mathrm{e}-03)$, T-bet $(\mathrm{r}=0.154 ; \mathrm{P}=8.20 \mathrm{e}-03)$, and $\mathrm{CD} 4$ $(\mathrm{r}=0.195, \mathrm{P}=8.16 \mathrm{e}-04)$, Th2 marker, GATA3 $(\mathrm{r}=$ $0.135, \mathrm{P}=2.08 \mathrm{e}-02)$, STAT6 $(\mathrm{r}=0.328, \mathrm{P}=8.53 \mathrm{e}-09)$, STAT5A $(\mathrm{r}=0.273, \mathrm{P}=2.11 \mathrm{e}-06)$, CCR4 $(\mathrm{r}=0.333$, $\mathrm{P}=5.02 \mathrm{e}-09)$, and Treg marker TGF $\beta(\mathrm{r}=0.194, \mathrm{P}$ $=8.65 \mathrm{e}-04)$, STAT5B $(\mathrm{r}=0.336, \mathrm{P}=3.54 \mathrm{e}-09)$, and CCR8 $(\mathrm{r}=0.279, \mathrm{P}=1.20 \mathrm{e}-06)$. ZBTB7A expression did not show any significant correlation with the expression of marker genes for CD8 $+\mathrm{T}$ cells, B cells, TAM and Th17 cells in UCEC. These findings suggest a relation between ZBTB7A expression and immune infiltration.

ZBTB7A represses E2F4 promoter activities in endometrial cancer cells

Analysis of ZBTB7A networks for transcription factor targets in UCEC, We found that ZBTB7A was associated with 
Table 2 Correlation analysis between ZBTB7A and related markers of immune cells

\begin{tabular}{|c|c|c|c|}
\hline \multirow[t]{2}{*}{ Description } & \multirow[t]{2}{*}{ Gene marker } & \multicolumn{2}{|c|}{ ZBTB7A (Purity) } \\
\hline & & Cor & $\mathbf{P}$ \\
\hline \multirow[t]{2}{*}{$\mathrm{CD}^{+} \mathrm{T}$ cell } & CD8A & 0.175 & $* *$ \\
\hline & CD8B & 0.105 & 0.074 \\
\hline \multirow{3}{*}{ T cell (general) } & CD3D & 0.139 & $* *$ \\
\hline & CD3E & 0.215 & $* * *$ \\
\hline & CD2 & 0.167 & $* *$ \\
\hline \multirow[t]{2}{*}{ B cell } & CD79A & 0.121 & * \\
\hline & CD19 & 0.042 & 0.471 \\
\hline \multirow[t]{3}{*}{ TAM } & CD68 & 0.22 & $* * *$ \\
\hline & CCL2 & -0.05 & 0.395 \\
\hline & IL 10 & -0.004 & 0.945 \\
\hline \multirow[t]{3}{*}{ Neutrophils } & CD66b (CEACAM8) & 0.309 & $* * *$ \\
\hline & CD11b (ITGAM) & 0.294 & $* * *$ \\
\hline & CCR7 & 0.222 & $* * *$ \\
\hline \multirow[t]{7}{*}{ Dendritic cell } & HLA-DPB1 & 0.212 & $* * *$ \\
\hline & HLA-DQB1 & 0.134 & * \\
\hline & HLA-DRA & 0.255 & $* * *$ \\
\hline & HLA-DPA1 & 0.238 & $* * *$ \\
\hline & BDCA1 (CD1C) & 0.246 & $* * *$ \\
\hline & BDCA4 (NRP1) & 0.226 & $* * *$ \\
\hline & CD11c (ITGAX) & 0.259 & $* * *$ \\
\hline \multirow[t]{5}{*}{ Th1 } & STAT4 & 0.166 & $* *$ \\
\hline & T-bet (TBX21) & 0.154 & $* *$ \\
\hline & CD4 & 0.195 & $* * *$ \\
\hline & TNF-a (TNF) & 0.041 & 0.484 \\
\hline & IFN- $\gamma($ IFNG $)$ & 0.083 & 0.159 \\
\hline \multirow[t]{6}{*}{ Th2 } & GATA3 & 0.135 & * \\
\hline & STAT6 & 0.328 & $* * *$ \\
\hline & STAT5A & 0.273 & $* * *$ \\
\hline & CCR4 & 0.333 & $* * *$ \\
\hline & IL13 & 0.066 & 0.259 \\
\hline & CXCR4 & -0.039 & 0.508 \\
\hline \multirow[t]{2}{*}{ Th17 } & STAT3 & 0.459 & $* * *$ \\
\hline & IL17A & 0.059 & 0.317 \\
\hline \multirow[t]{3}{*}{ Treg } & TGF $\beta$ (TGFB1) & 0.194 & $* * *$ \\
\hline & STAT5B & 0.336 & $* * *$ \\
\hline & CCR8 & 0.279 & $* * *$ \\
\hline
\end{tabular}

TAM, tumor-associated macrophage; Th: T helper cell; Treg, regulatory T cell; Cor, $R$ value of Spearman's correlation; Purity, correlation adjusted by purity. ${ }^{*} \mathrm{P}<0.05$, ${ }^{* * P}<0.01,{ }^{* * *} \mathrm{P}<0.001$
E2F4. Therefore, we chose Ishikawa cells to further verify the detected results from bioinformatics information. qRTPCR demonstrated that ZBTB7A overexpression repressed E2F4 mRNA levels in Ishikawa cells (Fig. 7a). Luciferase assay indicated the E2F4 promoter $(-2000 \sim+100)$ was significantly inactivated by ZBTB7A overexpression (Fig. 7b). Three potential ZBTB7A binding elements with E2F4 promoter from bioinformatics analysis (Fig. 7c-d). To examine which part was necessary for ZBTB7A mediated E2F4 expression, three predicted ZBTB7A binding sites from JASPAR database were deleted respectively. We found ZBTB7A nearly lose the ability to inhibit E2F4 transcriptional activity without the Del-E1 element (Fig. 7e), which implied the essence of E1 element for ZBTB7A binding to repress E2F4 transcription. To support our findings, chromatin immunoprecipitation (ChIP) assay was performed for E1 element. As shown in Fig. 7f, ZBTB7A could bind to E2F4 promoter and were enriched in the E1 region $(-483 \sim-471 \mathrm{bp})$.

\section{ZBTB7A inhibits proliferation and migration of endometrial} cancer cells.

* Next, we explored whether ZBTB7A affects biological ** function of endometrial cancer cells. ZBTB7A mRNA level ** $\quad$ was upregulated in Ishikawa cells after transfection with * pcDNA4-ZBTB7A plasmid (Fig. 8a). CCK-8 assay dem* $\quad$ onstrated that upregulated ZBTB7A notablely suppressed ** proliferation of Ishikawa cells (Fig. 8b). Furthermore, 0484 Migration assay revealed that overexpression of ZBTB7A 159 attenuated the ability of migration (Fig. 8c).

\section{Disscusion}

*** ZBTB7A is an essential factor in regulating different ${ }_{* * *}$ aspects of human cancer. To understand more detail 259 potential function and regulatory network of ZBTB7A in 0.508 UCEC, we conducted the bioinformatics analysis of public ${ }_{* * *}$ databases to provide future research of UCEC. This study 317 shows that ZBTB7A mRNA levels are markedly down** regulated in the Endometrial Serous Adenocarcinoma, E* Endometrial Mixed Adenocarcinoma, and Endometrial * Endometrioid Adenocarcinoma of oncomine datasets. Low ZBTB7A mRNA expression not merely correlates with unfavorable prognosis in UCEC, but also in CHOL and HNSC-HPV cancers, Furthermore, There exist a markedly relationshiop between ZBTB7A and chlinical characteristics for age, race, and tumor stage in UCEC. There

\footnotetext{
(See figure on next page.)

Fig. 6 Correlation of ZBTB7A expression and the marker genes of infiltrating immune cells in UCEC (TIMER). The scatter plots showed correlation between ZBTB7A expression and the gene markers of (a) CD8 ${ }^{+}$T cell (CD8A); (b) T cell (general) (CD3D, CD3E, and CD2); (c) B cell (CD79A); (d) TAM (CD68); (e) Th17 (STAT3); (f) Neutrophils (CD66b, CD1 1b, and CCR7); (g, h) Dendritic cell (HLA-DPB1, HLA-DQB1, HLA-DRA, HLA-DPA1, BDCA1, BDCA4 and CD11C); (i) Th1 (STAT4, T-bet, and CD4); (j) Th 2 (GATA3, STAT6, STAT5A, and CCR4); (k) and Treg (TGFB1, STAT5B, and CCR8) in UCEC samples ( $\mathrm{n}=$ 545)
} 

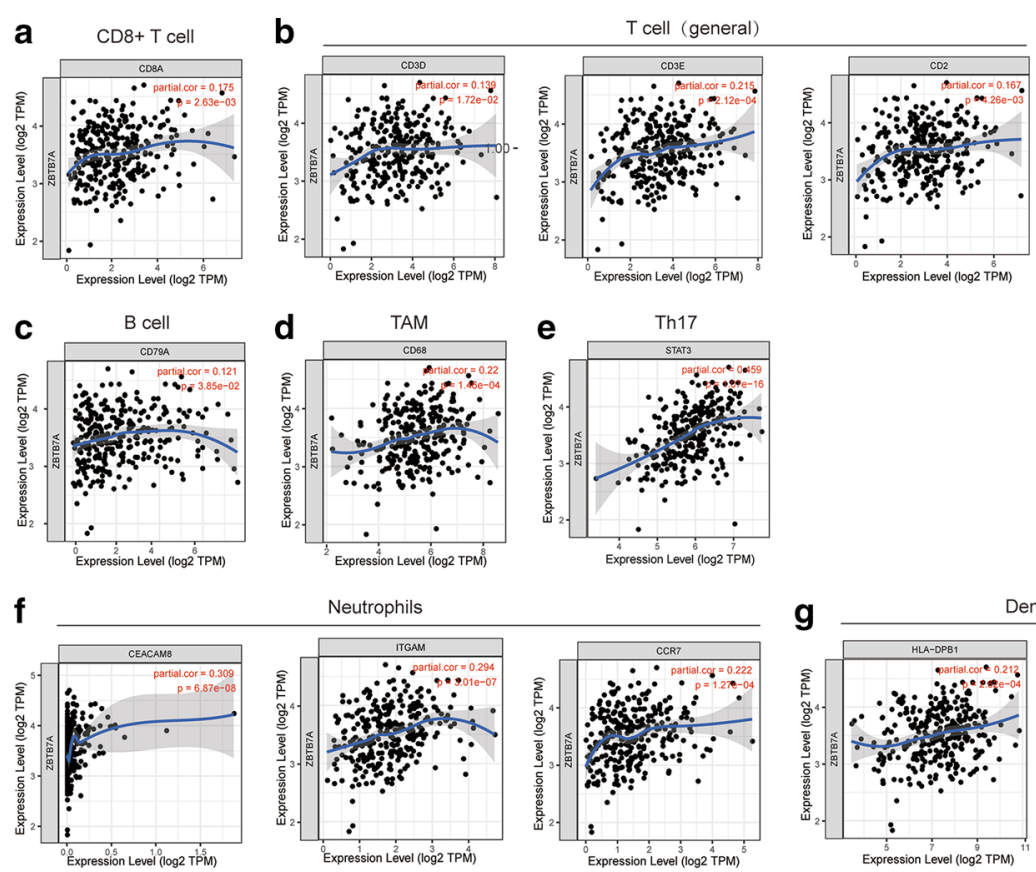

Neutrophils
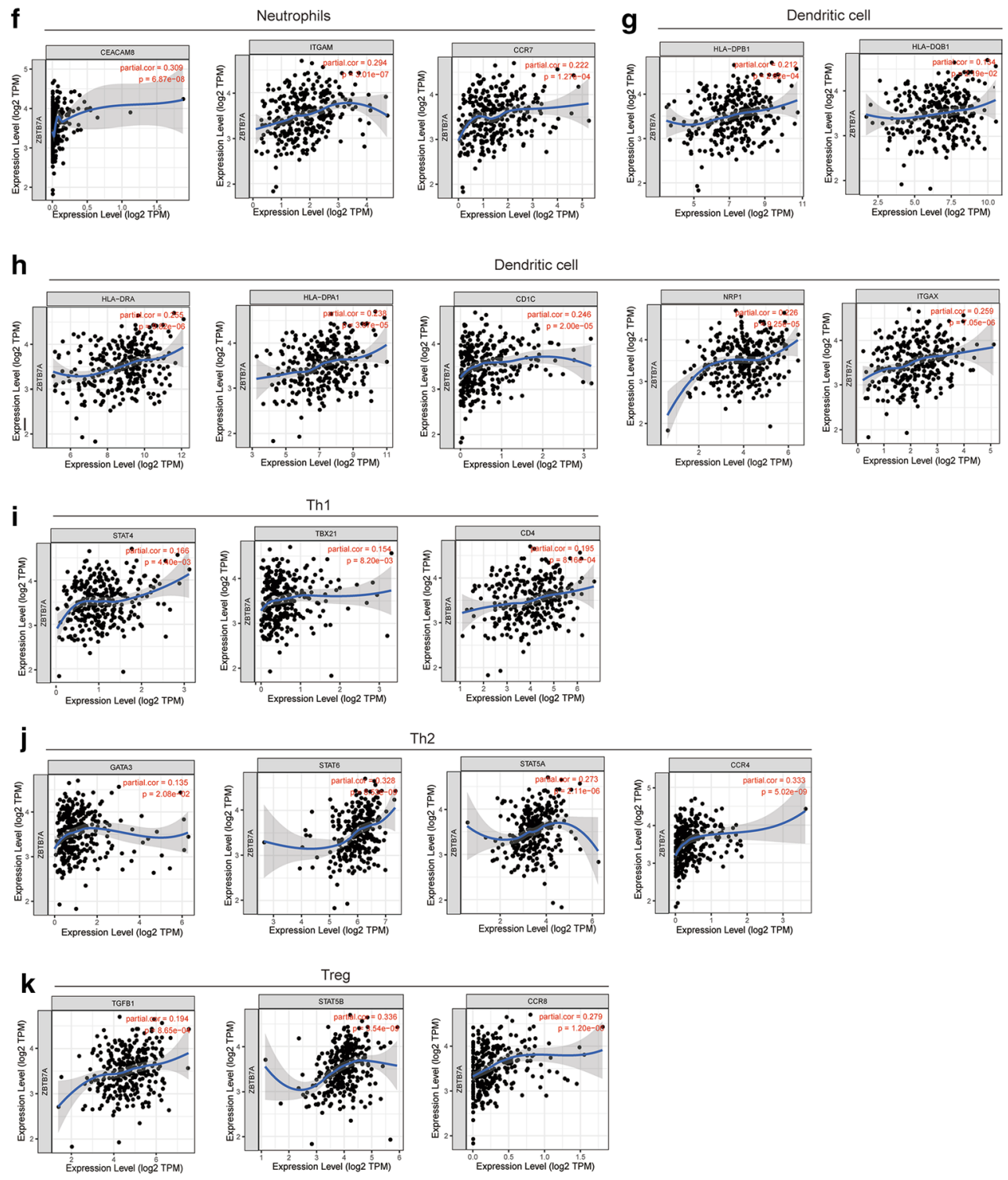


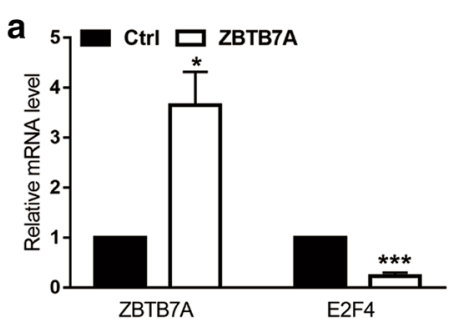

C
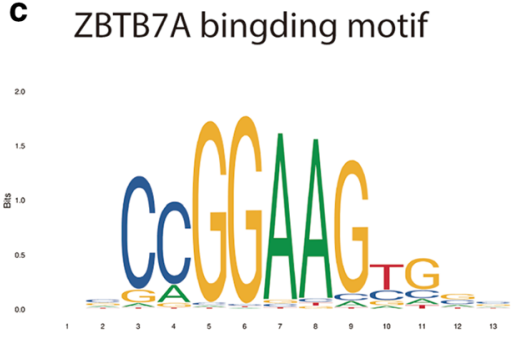

e

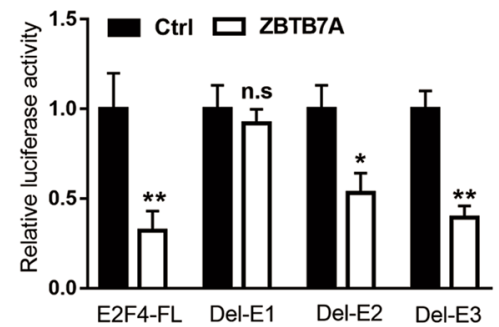

b

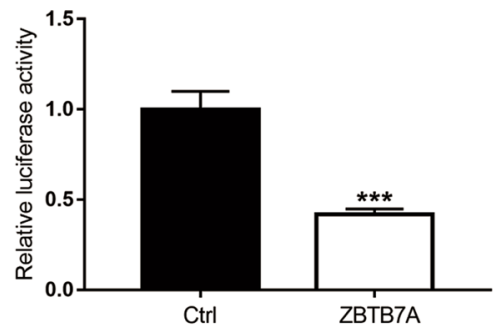

d

\section{E2F4 promoter}

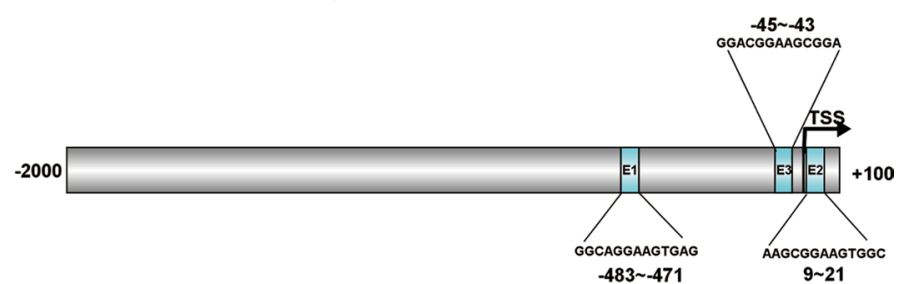

f

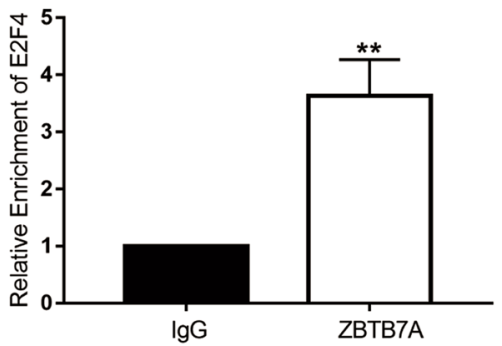

Fig. 7 ZBTB7A can repress E2F4 transcription in endometrial cancer cells. a E2F4 mRNA levels with ZBTB7A overexpression in Ishikawa cells. b E2F4 promoter transcriptional activity was detected by Luciferase assay when cells were transfected with $300 \mathrm{ng}$ ZBTB7A plasmid. c Schematic diagram of ZBTB7A binding motif from JASPAR Database. $\mathbf{d}$ Three prospected ZBTB7A responsive elements (E1, E2, and E3) in the E2F4 promoter part. TSS represents the transcriptional start site of E2F4. e Three potential ZBTB7A binding sites of the E2F4 promoter was eliminated respectively and named Del-E1, Del-E2, and Del-E3. The transcriptional activity of three depleted promoter were examined by Luciferase assay when ZBTB7A plasimid was transfected into Ishikawa cells. $\mathbf{f}$ Chromatin immunoprecipitation (ChIP) assay revealed that ZBTB7A might bind to the E1 part of E2F4 promoter in Ishikawa. The values are presented as the means \pm SD from three independent experiments. ${ }^{*} P<0.05,{ }^{* *} P<0.01,{ }^{* * *} P<0.001$

was variability in the expression of ZBTB7A in different types of cancers, which may reflect differences in the data collection methods and the underlying causative mechanisms. However, the ZBTB7A expression data was consistent in UCEC tissues across various databases. These data strongly imply that ZBTB7A is a potential prognostic biomarker in UCEC. Somatic copy number variation (CNVs) often occurs in human tumors. CNVs can change the content of the genome and phenotypic differences, which may provide potential prognostic information. Our studies detect the copy number of ZBTB7A is increased and that the dominant type of ZBTB7A alteration is deep deletion, which should be responsible for the shorter survival in UCEC.

To probe the signaling events in controlling abnormal ZBTB7A expression, we tested the ZBTB7A coexpression network. Data suggest that the functional consequence of ZBTB7A mainly include pri-miRNA transcription by RNA polymerase II, negative regulation of production of miRNAs involved in gene silencing by miRNA, regulation of autophagy. These findings are consistent with the molecular pathways that result in UCEC.

We further found that ZBTB7A in UCEC is associated with a network of kinases, including CDK1, PLK1, AURKB, ATM, and CDK2. These kinases can regulate the cell cycle and mitosis, and showed differential expression, except CDK2 in UCEC. PKL1 is the primary regulator of the cell cycle [23]. In the cell cycle, PLK1 controls mitotic entry and the G2/M checkpoint [24]. AURKB plays a central role in mitosis and participates in the division of cells from G2 phase to $M$ phase [25, 26]. The expression of the mitotic cell cycle (CDK1) was significantly suppressed by IRAK1 knockdown [27]. In UCEC, ZBTB7A may regulate cell cycle progression via interacted kinases. 


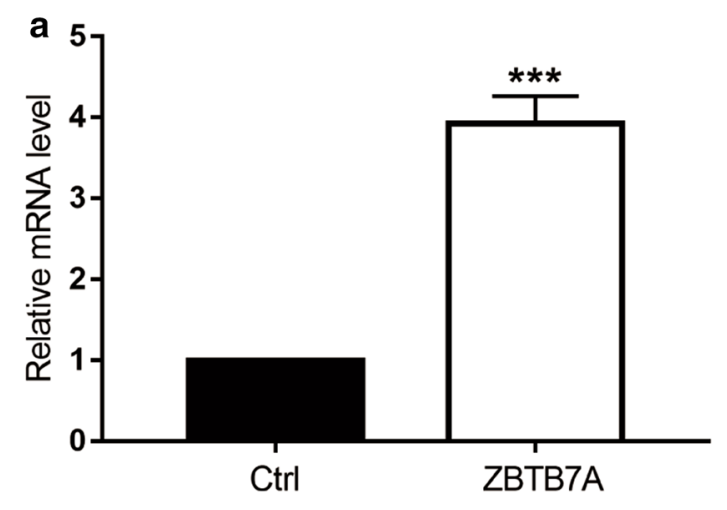

C

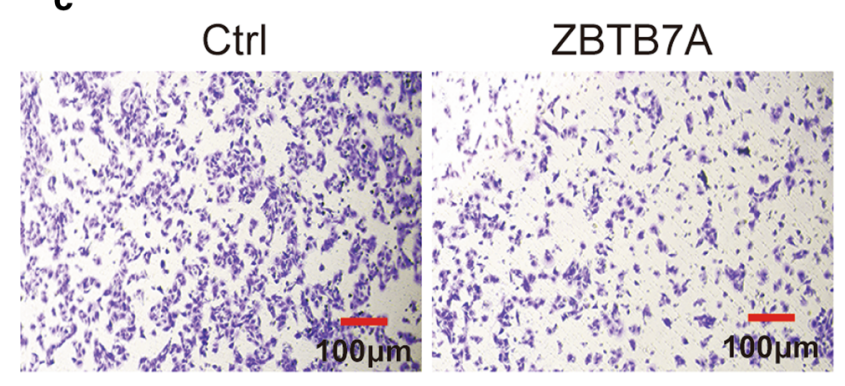

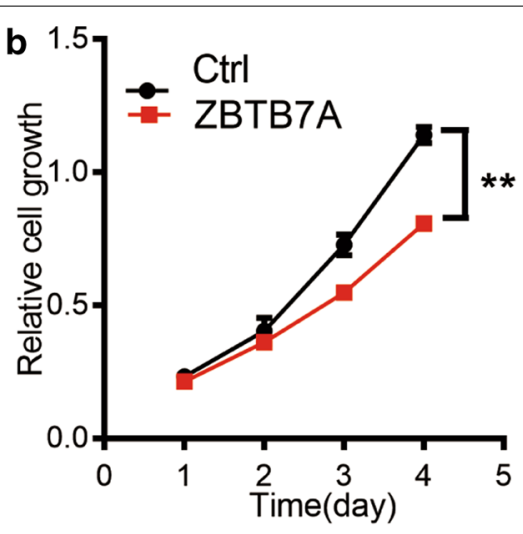

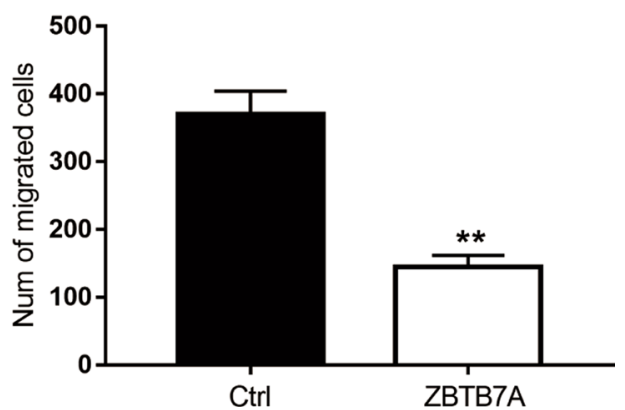

Fig. 8 ZBTB7A inhibits proliferation and migration of Ishikawa cells. a The effect of plasmid pcDNA4-ZBTB7A overexpressed in Ishikawa cells. ZBTB7A inhibits growth (b), and migration (c) in Ishikawa cells

MicroRNAs (miRNAs) are small non-coding RNAs that often serve critical roles in human diseases. Our study identified several miRNAs that were associated with ZBTB7A. The expression of miR-212/132 may be related to the regulation of hormones and metabolism [28]. Low expression of miR-202 is associated with poor prognosis of UCEC [29]. miR-522 stimulated the progression of endometrial carcinoma by inhibiting MAOB [30]. miR499 and miR-485-3P are associated with increased risk of cancer [31, 32]. We also found that the network of transcription factors targeted by ZBTB7A was E2F4. The role of E2F4 in the differentiation of various tissues is mainly to regulate non-cell cycle genes [33], which can directly inhibit apoptosis genes [34] and promote tumor growth by protecting cancer cells from death. We used Ishikawa cells to further verify the results that ZBTB7A inhibits the transcriptional level of E2F4 at the site of - 483 -471 promoter and predicted that TPX2 and TTK might be the target of E2F4, which needs to be further verified in the next studies. Furthermore, increased ZBTB7A expression repress Ishikawa cells proliferation and migration obviously. Our results suggest that ZBTB7A might act through this factor to regulate cell function of UCEC.

ZBTB7A mRNA levels correlate with the different immune cell type markers in UCEC. This study demonstrates that there is a strong positive correlation between ZBTB7A expression with the infiltration of CD8 + T cells, neutrophils, and dendritic cells. This suggests that ZBTB7A plays a vital role in regulating tumor immunity, and therefore influences UCEC prognosis. We observed correlation between the levels of ZBTB7A mRNA and the expression of the $T$ cell (general), Th1, Th2, Treg, Neutrophils, and Dendritic cell, which indicates that ZBTB7A regulates infiltration and activity of Neutrophils, and Dendritic cell. ZBTB7A expression also displays association with the markers of different subsets of $\mathrm{T}$ helper (Th) cells, including $\mathrm{T}$ cell (general) (CD2, CD3D, and CD3E), Th1 (T-bet, STAT-4, IFN- $\gamma$ and TNF- $\alpha$ ), Th2 (GATA3, STAT6, STAT5A, CCR4, IL13, and CXCR4), and Tregs (TGF $\beta$, STAT5B and CCR8). Above all demonstrate the function for ZBTB7A in regulating tumor-infiltration of T-helper cells.

\section{Conclusion}

Our study systematically applied public database to guide the research of ZBTB7A in endometrial cancer. Our work determine that ZBTB7A is notably downregulated in UCEC, inhibits proliferation and migration in endometrial cancer cells, serves as an indicator for favorable prognosis and immune infiltration. Besides, 
we verify that ZBTB7A can repress the transcription of E2F4, which may be responsible for function of ZBTB7A in endometrial cancer. In Sum, ZBTB7A could be identified as an important potential biomarker for endometrial cancer.

\section{Supplementary information}

Supplementary information accompanies this paper at https://doi. org/10.1186/s12935-020-01600-5.

Additional file1: Fig. S1. ZBTB7A mRNA level was associated with the survival in $\mathrm{CHOL}$ and HNSC-HPV pos.

Additional file 2: Fig. S2. Expression and survival outcome of ZBTB7Arelated regulators. Top 5 kinase regulators of ZBTB7A co-expressed genes. PLK1, CDK1, and AURKB were significantly higher expressed in tumor tissues, ATM was dramatically lower expressed in tumor tissues, except CDK2. In addition, all these kinase genes were not significantly associated with the overall survival of UCEC.

Additional file 3: Fig. S3. Correlation of ZBTB7A expression with immune infiltration levels in 9 cancers in the TIMER database.

Additional file 4: Fig. S4. ZBTB7A CNV affecting the distribution in various immune cells in 9 cancers in the TIMER database.

\section{Acknowledgements}

Not applicable

\section{Authors' contributions}

RG: Conceptualization (equal); Data curation (equal); Investigation (lead); Writing-original draft (lead); Writing-review \& editing (lead); Funding acquisition (lead). YHZ: Data curation (equal); Formal analysis (equal); Investigation (lead); Writing-original draft (lead); Writing-review \& editing (lead); DHZ: Data curation (equal); Formal analysis (equal); Investigation (equal). QDL: Investigation (equal). RML: Conceptualization (lead); Project administration (lead); Supervision (lead). XLG: Conceptualization (lead); Project administration (lead); Supervision (lead). All authors read and approved the final manuscript.

\section{Funding}

This study was supported by the funding from National Natural Science Foundation of China [81901453], Science and Technology Planning Project of Foshan City of China [2018AB000281].

\section{Availability of data and materials}

The data that support the findings of this study are available from the corresponding author upon reasonable request.

\section{Ethics approval and consent to participate} Not applicable.

\section{Consent for publication}

Not applicable.

\section{Competing interests}

The authors confirm that there are no conflicts of interest.

\footnotetext{
Author details

1 Department of Gynecology, Affiliated Foshan Maternity \& Child Healthcare Hospital, Southern Medical University, Foshan 52800, China. ${ }^{2}$ Department of Gynecology and Obstetrics, The First Affiliated Hospital, Jinan University, Guangzhou 510632, China. ${ }^{3}$ Foshan Maternal and Children Healthy Research Institute, Affiliated Foshan Maternity \& Child Healthcare Hospital, Southern Medical University, Foshan, China. ${ }^{4}$ Department of Pathology, Affiliated Foshan Maternity \& Child Healthcare Hospital, Southern Medical University, Foshan, China.
}

Received: 19 August 2020 Accepted: 6 October 2020

Published online: 09 November 2020

\section{References}

1. Bray F, Ferlay J, Soerjomataram I, Siegel RL, Torre LA, Jemal A. Global cancer statistics 2018: GLOBOCAN estimates of incidence and mortality worldwide for 36 cancers in 185 countries. CA. 2018;68(6):394-424.

2. Chen Y, Huang Q, Chen Q, Lin Y, Sun X, Zhang H, Zhu M, Dong S. The inflammation and estrogen metabolism impacts of polychlorinated biphenyls on endometrial cancer cells. Toxicol Vitro. 2015;29(2):308-13.

3. Ouyang D, Li R, Li Y, Zhu X. Construction of a competitive endogenous RNA network in uterine corpus endometrial Carcinoma. Med Sci Monitor. 2019;25:7998-8010.

4. Liu C, Zhang YH, Deng Q, Li Y, Huang T, Zhou S, Cai YD. Cancer-related triplets of mRNA-IncRNA-miRNA revealed by integrative network in uterine corpus endometrial carcinoma. Biomed Res Int. 2017;2017:3859582.

5. Jiang L, Siu MK, Wong OG, Tam KF, Lam EW, Ngan HY, Le XF, Wong ES, Chan HY, Cheung AN. Overexpression of proto-oncogene FBI-1 activates membrane type 1-matrix metalloproteinase in association with adverse outcome in ovarian cancers. Mol Cancer. 2010;9:318.

6. Zu X, Ma J, Liu H, Liu F, Tan C, Yu L, Wang J, Xie Z, Cao D, Jiang Y. Prooncogene Pokemon promotes breast cancer progression by upregulating survivin expression. Breast cancer Res. 2011;13(2):R26.

7. Ramos Pittol JM, Oruba A, Mittler G, Saccani S, van Essen D. Zbtb7a is a transducer for the control of promoter accessibility by NF-kappa B and multiple other transcription factors. PLoS Biol. 2018;16(5):e2004526.

8. Molloy ME, Lewinska M, Williamson AK, Nguyen TT, Kuser-Abali G, Gong L, Yan J, Little JB, Pandolfi PP, Yuan ZM. ZBTB7A governs estrogen receptor alpha expression in breast cancer. J Mol Cell Biol. 2018;10(4):273-84.

9. Zhang YQ, Xiao CX, Lin BY, Shi Y, Liu YP, Liu JJ, Guleng B, Ren JL. Silencing of Pokemon enhances caspase-dependent apoptosis via fas- and mitochondria-mediated pathways in hepatocellular carcinoma cells. PLoS ONE. 2013;8(7):e68981.

10. Bezzi M, Seitzer N, Ishikawa T, Reschke M, Chen M, Wang G, Mitchell C, Ng C, Katon J, Lunardi A, et al. Diverse genetic-driven immune landscapes dictate tumor progression through distinct mechanisms. Nat Med. 2018:24(2):165-75.

11. Liu XS, Liu Z, Gerarduzzi C, Choi DE, Ganapathy S, Pandolfi PP, Yuan ZM. Somatic human ZBTB7A zinc finger mutations promote cancer progression. Oncogene. 2016;35(23):3071-8.

12. Tang Z, Li C, Kang B, Gao G, Li C, Zhang Z. GEPIA: a web server for cancer and normal gene expression profiling and interactive analyses. Nucleic Acids Res. 2017;45(W1):W98-w102.

13. Chandrashekar DS, Bashel B, Balasubramanya SAH, Creighton CJ, PonceRodriguez I, Chakravarthi B, Varambally S. UALCAN: a portal for facilitating tumor subgroup gene expression and survival analyses. Neoplasia. 2017;19(8):649-58.

14. Gao J, Aksoy BA, Dogrusoz U, Dresdner G, Gross B, Sumer SO, Sun Y, Jacobsen A, Sinha R, Larsson E, et al. Integrative analysis of complex cancer genomics and clinical profiles using the cBioPortal. Sci Signal. 2013;6(269):pl1.

15. Vasaikar SV, Straub P, Wang J, Zhang B. LinkedOmics: analyzing multiomics data within and across 32 cancer types. Nucleic Acids Res. 2018;46(D1):D956-d963963.

16. Li T, Fan J, Wang B, Traugh N, Chen Q, Liu JS, Li B, Liu XS. TIMER: a web server for comprehensive analysis of tumor-infiltrating immune cells. Can Res. 2017;77(21):e108-e110110.

17. Li B, Severson E, Pignon JC, Zhao H, Li T, Novak J, Jiang P, Shen H, Aster JC, Rodig S, et al. Comprehensive analyses of tumor immunity: implications for cancer immunotherapy. Genome Biol. 2016;17(1):174.

18. Szklarczyk D, Morris JH, Cook H, Kuhn M, Wyder S, Simonovic M, Santos A, Doncheva NT, Roth A, Bork P, et al. The STRING database in 2017: quality-controlled protein-protein association networks, made broadly accessible. Nucleic Acids Res. 2017;45(D1):D362-d368368.

19. Tripathi S, Pohl MO, Zhou Y, Rodriguez-Frandsen A, Wang G, Stein DA, Moulton HM, DeJesus P, Che J, Mulder LC, et al. Meta- and orthogonal integration of influenza "OMICs" data defines a role for UBR4 in virus budding. Cell Host Microbe. 2015;18(6):723-35. 
20. Geng R, Tan X, Wu J, Pan Z, Yi M, Shi W, Liu R, Yao C, Wang G, Lin J, et al. RNF183 promotes proliferation and metastasis of colorectal cancer cells via activation of NF-kappaB-IL-8 axis. Cell Death Dis. 2017;8(8):e2994.

21. Yang C, Shen S, Zheng X, Ye K, Ge H, Sun Y, Lu Y. Long non-coding RNA LINC00337 induces autophagy and chemoresistance to cisplatin in esophageal squamous cell carcinoma cells via upregulation of TPX2 by recruiting E2F4. FASEB J. 2020;34(5):6055-69.

22. Salvatore G, Nappi TC, Salerno P, Jiang Y, Garbi C, Ugolini C, Miccoli P, Basolo F, Castellone MD, Cirafici AM, et al. A cell proliferation and chromosomal instability signature in anaplastic thyroid carcinoma. Can Res. 2007;67(21):10148-58.

23. Zitouni S, Nabais C, Jana SC, Guerrero A, Bettencourt-Dias M. Polo-like kinases: structural variations lead to multiple functions. Nat Rev Mol Cel Biol. 2014;15(7):433-52

24. Colicino EG, Hehnly $H$. Regulating a key mitotic regulator, polo-like kinase 1 (PLK1). Cytoskeleton. 2018;75(11):481-94.

25. Carmena M, Earnshaw WC. The cellular geography of aurora kinases. Nat Rev Mol Cell Biol. 2003;4(11):842-54.

26. Goldenson B, Crispino JD. The aurora kinases in cell cycle and leukemia. Oncogene. 2015;34(5):537-45.

27. Wang Y, Wang Y, Duan X, Wang Y, Zhang Z. Interleukin-1 receptor-associated kinase 1 correlates with metastasis and invasion in endometrial carcinoma. J Cell Biochem. 2018;119(3):2545-55.

28. Wanet A, Tacheny A, Arnould T, Renard P. miR-212/132 expression and functions: within and beyond the neuronal compartment. Nucleic Acids Res. 2012;40(11):4742-53
29. Deng X, Hou C, Liang Z, Wang H, Zhu L, Xu H. miR-202 suppresses cell proliferation by targeting FOXR2 in endometrial adenocarcinoma. Dis Markers. 2017;2017:2827435.

30. Zhang HC, Han YY, Zhang XM, Xiao N, Jiang T, Zhu S, Wang EP, Chen CB. miR-522 facilitates the prosperities of endometrial carcinoma cells by directly binding to monoamine oxidase B. Kaohsiung J Med Sci. 2019:35(10):598-606.

31. Xu Z, Zhang E, Duan W, Sun C, Bai S, Tan X. The association between miR-499 polymorphism and cancer susceptibility: a meta-analysis. OncoTargets Therapy. 2015;8:2179-86.

32. Zhang Y, Sui R, Chen Y, Liang H, Shi J, Piao H. Downregulation of miR485-3p promotes glioblastoma cell proliferation and migration via targeting RNF135. Exp Therap Med. 2019;18(1):475-82.

33. Tseng YH, Butte AJ, Kokkotou E, Yechoor VK, Taniguchi CM, Kriauciunas KM, Cypess AM, Niinobe M, Yoshikawa K, Patti ME, et al. Prediction of preadipocyte differentiation by gene expression reveals role of insulin receptor substrates and necdin. Nat Cell Biol. 2005;7(6):601-11.

34. Dingar D, Konecny F, Zou J, Sun X, von Harsdorf R. Anti-apoptotic function of the E2F transcription factor 4 (E2F4)/p130, a member of retinoblastoma gene family in cardiac myocytes. J Mol Cell Cardiol. 2012;53(6):820-8

\section{Publisher's Note}

Springer Nature remains neutral with regard to jurisdictional claims in published maps and institutional affiliations.
Ready to submit your research? Choose BMC and benefit from:

- fast, convenient online submission

- thorough peer review by experienced researchers in your field

- rapid publication on acceptance

- support for research data, including large and complex data types

- gold Open Access which fosters wider collaboration and increased citations

- maximum visibility for your research: over 100M website views per year

At BMC, research is always in progress.

Learn more biomedcentral.com/submissions 